\title{
A new complex allele of the CFTR gene partially explains the variable phenotype of the L997F mutation
}

\author{
Marco Lucarelli, BS, PhD ${ }^{I}$, Lorena Narzi, BS, PhD ${ }^{2}$, Silvia Pierandrei, BS, $P h D^{I}$, \\ Sabina Maria Bruno, BS, $P h D^{l}$, Antonella Stamato, $B S^{2}$, Miriam d'Avanzo, $M D, P h D^{3}$, \\ Roberto Strom, $M D, P h D^{l}$, and Serena Quattrucci, $M D, P h D^{2}$
}

\begin{abstract}
Purpose: To evaluate the role of complex alleles, with two or more mutations in cis position, of the cystic fibrosis transmembrane conductance regulator (CFTR) gene in the definition of the genotype-phenotype relationship in cystic fibrosis (CF), and to evaluate the functional significance of the highly controversial L997F CFTR mutation. Methods: We evaluated the diagnosis of CF or CFTR-related disorders in 12 unrelated subjects with highly variable phenotypes. According to a first CFTR mutational analysis, subjects appeared to be compound heterozygotes for a classic mutation and the L997F mutation. A further CFTR mutational analysis was conducted by means of a protocol of extended sequencing, particularly suited to the detection of complex alleles. Results: We detected a new [R117L; L997F] CFTR complex allele in the four subjects with the highest sweat test values and CF. The eight subjects without the complex allele showed the most varied biochemical and clinical outcome and were diagnosed as having mild CF, CFTR-related disorders, or even no disease. Conclusions: The new complex allele partially explains the variable phenotype in CF subjects with the L997F mutation. CFTR complex alleles are likely to have a role in the definition of the genotype-phenotype relationship in CF. Whenever apparently identical CFTR-mutated genotypes are found in subjects with divergent phenotypes, an extensive mutational search is mandatory. Genet Med 2010:12(9):548-555.
\end{abstract}

Key Words: CFTR, complex alleles, cystic fibrosis, genotype-phenotype relationship, mutational search

Costi ystic fibrosis (CF) is a recessive autosomal disease caused by cystic fibrosis transmembrane conductance regulator (CFTR) gene (Genbank accession NG_016465) mutations. ${ }^{1,2}$ Clinical manifestations of CF vary to such an extent that some mono- or oligosymptomatic phenotypes have to be distinguished from polysymptomatic classic $\mathrm{CF}$, as occurs with CFTR-related disorders (CFTR-RD). ${ }^{3}$ It is generally accepted that the phenotypical severity of CF or CFTR-RD is essentially correlated with the residual function of the CFTR gene, ${ }^{4}$ which is produced by intragenic variability due to the large number of mutations and to the even larger number of combinations of these mutations. However, the influence of modifier genes as a source of extragenic variability on the final phenotype has been

From the ${ }^{1}$ Department of Cellular Biotechnologies and Hematology, ${ }^{2}$ Cystic Fibrosis Center of Lazio Region, Department of Pediatrics, and ${ }^{3}$ Pediatric Radiology Service, Department of Pediatrics, Sapienza University of Rome, Rome, Italy.

Marco Lucarelli, Sez. Biochimica Clinica, Dip. Biotecnologie Cellulari ed Ematologia, c/o Azienda Policlinico Umberto I, Viale Regina Elena 324, Roma 00161, Italia. E-mail: marco.lucarelli@uniroma1.it.

Disclosure: The authors declare no conflict of interest

Submitted for publication March 31, 2010.

Accepted for publication June 1, 2010

Published online ahead of print August 11, 2010.

DOI: $10.1097 /$ GIM.0b013e3181ead634 observed $^{5}$ when a high phenotypical variability occurs in subjects with identical CFTR-mutated genotypes. An aspect of the genotype-phenotype relationship that has not yet been fully addressed is the involvement of complex alleles as a further source of CFTR genetic variability. An allele is called complex when it carries two (or more) mutations in cis. The most widely used protocols for a mutational search within the CFTR gene, whether they be protocols based on mutational panels including well-characterized classic CFTR mutations or gene scanning protocols, are designed in such a way as to stop when two mutations on different alleles are found. Thus, additional mutations that may be present in cis with the already found mutations may escape detection. Because of this common limitation of mutational search protocols, the mutated genotypes of CFaffected subjects with varying clinical presentations may appear identical despite the presence of unrevealed complex alleles that might explain the divergent phenotypes. As regards the consequences of an unrevealed complex allele, a distinction must be made between finding the two-CFTR mutations already known to cause disease on both alleles, which may result in an unclear genotype-phenotype relationship, and finding one classic mutation on one allele and one sequence variation with little or conflicting functional data on the other, which may instead result in diagnostic errors or misclassification of the sequence variation. Although the CFTR mutation L997F has been known since 1992, it is still highly controversial from the functional point of view, and its pathogenetic role has not yet been fully elucidated. L997F was initially believed to be a polymorphism ${ }^{6}$ then it was been reported to cause both CFTR-RD and no disease. The presence of L997F has been linked to pulmonary diseases, ${ }^{7,8}$ disseminated bronchiectasis,,${ }^{9,10}$ neonatal hypertrypsinaemia with normal sweat test, ${ }^{11-14}$ idiopathic pancreatitis, ${ }^{12,15-17}$ and congenital absence of vas deferens. ${ }^{18}$ It has also been suggested that L997F negatively influences the clinical course of primary sclerosis cholangitis. ${ }^{19}$ In subjects with idiopathic pancreatitis, the L997F CFTR mutation has also been found to be associated with mutations of the serine protease inhibitor Kazal type 1/pancreatic secretory trypsin inhibitor (SPINK1) gene, namely the N34S mutation ${ }^{20}$ and a deletion encompassing the entire gene. ${ }^{21}$ However, there is also evidence suggesting that homozygosity for L997F does not cause disease. ${ }^{22,23}$ The frequency of L997F in the general population has been reported to be $\sim 0.5 \%,{ }^{14,24}$ which is neither high enough to clearly exclude its pathogenetic role nor so low as to strongly support the pathogenetic hypothesis.

To shed light on the general issue of the role of complex alleles in the genotype-phenotype relationship in CF, we studied the functional and clinical significance of the L997F CFTR mutation. For this purpose, we studied 12 unrelated compound heterozygotes for a classic CFTR mutation on one allele and, according to a first CFTR mutational analysis, apparently only L997F on the other allele. These 12 subjects also had somewhat different phenotypes as assessed by biochemical and clinical studies. They underwent complete sequencing of all CFTR 
exons, adjacent intronic zones, and 5'-flanking regions to verify whether their phenotypic variability was linked to any additional CFTR sequence variations in cis with L997F.

\section{MATERIALS AND METHODS}

\section{Selected subjects and genetic testing cascade}

The study is a retrospective study that does not require ethics committee approval at our institution. Informed consent was obtained from subjects included.

We selected, from a larger group of subjects with a diagnosis or presumptive diagnosis of CF, 12 unrelated subjects who had a classic mutation and the L997F mutation in different alleles of the CFTR gene. In the analysis of the larger case series, the genetic testing cascade included a first-level genetic analysis using the CF-oligonucleotide ligation assay (OLA) mutational panel, a second-level genetic analysis using a multistepextended CFTR sequencing protocol (which analyzes successive groups of exons), and an analysis of the parents to verify allelic segregation (see also, "Mutational analysis" section). This mutational search was interrupted when two mutations in different alleles were found. The 12 subjects studied here instead underwent the extended CFTR mutational analysis described later even when two segregating mutations had previously been found. This mutational search protocol is more suited to the detection of complex alleles.

\section{Biochemical, microbiologic, and clinical characterization}

The subjects enrolled underwent: a sweat test, with a quantitative pilocarpine iontophoresis method, ${ }^{25}$ using the Macroduct device (Delcon, Italy) for sweat collection and the PCL M3 chloride analyzer Jenway (VWR International, Italy) for measurement; a pancreatic function evaluation by dosage of fecal elastase $1^{26}$ (using the pancreatic fecal elastase test; Meridian Bioscience, Italy); and a clinical examination. Nutritional status was evaluated by weight and height centiles. Liver disease was assessed by physical inspection, dosage of serum liver enzymes (aspartate aminotransferase, alanine aminotransferase, and $\gamma$-glutamyltransferase with common methods), and hepatopancreatic ultrasonography. Pulmonary status was evaluated by forced expiratory volume in the first second (FEV1) assessment (for subjects aged 8-years old, unless differently indicated) and chest radiographs according to the Brasfield scoring system. ${ }^{27}$ Microbiologic status was evaluated by bacteriologic analysis of oropharyngeal cultures by deep suctioning, using common and shared laboratory approaches. ${ }^{28} \mathrm{CF}$ and CFTR-RD diagnoses were performed according to current guidelines. ${ }^{3,29,30}$ Whenever applicable, semen analysis was performed according to World Health Organization (WHO) guidelines. ${ }^{31}$

According to the recent guidelines, ${ }^{30}$ the sweat test in subjects up to 6 months of age was considered negative if $<30$ $\mathrm{mEq} / \mathrm{L}$, pathologic if $\geq 60 \mathrm{mEq} / \mathrm{L}$, and borderline in the $30-59$ $\mathrm{mEq} / \mathrm{L}$ range; for all other subjects, the sweat test was considered negative if $<40 \mathrm{mEq} / \mathrm{L}$, pathologic if $\geq 60 \mathrm{mEq} / \mathrm{L}$, and borderline in the $40-59 \mathrm{mEq} / \mathrm{L}$ range. Although Table 1 only shows the mean value of repeated sweat test measurements (from two to four) on enrollment and during follow-up, the patients age was taken into account when they were classified as having a negative, borderline, or pathologic sweat test. We classified the severity of CF as follows: mild CF if FEV1 $>70 \%$ and Bransfield score $>15$; moderate $\mathrm{CF}$ if FEV1 ranged from $40 \%$ to $70 \%$ and Bransfield score $<15$; and severe CF if FEV1 $<40 \%$ and Bransfield score $<15$.

\section{Mutational analysis}

DNA was extracted from peripheral blood using the QIAamp DNA blood midi kit (QIAGEN, Hilden, Germany). The CFOLA assay (Abbott, Wiesbaden, Germany), ${ }^{32}$ including the 32 most common mutations of the CFTR gene worldwide, was used as a first-level genetic analysis. A 96-well formatted method for the sequencing of the $5^{\prime}$-flanking region, all exons, and the adjacent intronic regions of the CFTR gene was used as a second-level genetic analysis. ${ }^{33}$ The $(\mathrm{TG})_{m} \mathrm{~T}_{n}$ polymorphic tract in the CFTR intron 8 was studied by sequencing, as previously described. ${ }^{34}$ The BigDye Terminator version 1.1 Cycle Sequencing kit (Applied Biosystems, Foster City, CA) was used for sequencing. The first-level OLA products and the second-level sequences were run by an ABI PRISM 3100 Avant Genetic Analyzer (Applied Biosystems). Electropherograms were analyzed by specific Genotyper (for OLA) and Seqscape (for sequencing) templates. Segregation of the mutations found was always verified by analysis of the parents. The CFTR molecular genetic data were interpreted and are reported according to the updated European recommendations. ${ }^{3}$

The state of conservation of both the L997 and R117 aminoacidic residues was studied by comparing the following 10 species using CLUSTALW software: human, mouse, rat, macaque, bovine, sheep, pig, dog, rabbit, and xenopus.

\section{Statistical analysis}

The comparison between the mean sweat test values of the groups with or without the complex allele was performed by means of the Student $t$-test, using the data analysis tool of Excel software (Microsoft, Redmond, WA).

\section{RESULTS}

\section{Genetic analysis, biochemical features of the mutations found, and state of conservation of L997F and $\mathrm{R} 117 \mathrm{~L}$ residues}

Five different CFTR mutations were found (those included in the CF-OLA panel were confirmed by sequencing) on one allele of the subjects analyzed (Table 1). F508del (c.1520_ 1522delTCT), the most common CF mutation worldwide, is a trinucleotide deletion resulting in the loss of phenylalanine in position 508 of the CFTR protein in the cytoplasmic first nucleotide-binding domain. W1282X (c.3846G $>$ A) is a nonsense mutation that produces a truncated CFTR form. G85E (c.254G >A), S549R(A >C) (c.1645A >C), and L320V (c.958T > $\mathrm{G})$ are missense mutations located in the first membrane spanning domain-first transmembrane segment, in the first nucleotide-binding domain, and in the first membrane spanning domainfifth transmembrane segment, respectively. F508del, W1282X, $\mathrm{G} 85 \mathrm{E},{ }^{35}$ and $\mathrm{S} 549 \mathrm{R}(\mathrm{A}>\mathrm{C})^{36}$ are considered classic CFTR mutations that cause a severe $\mathrm{CF}$ form when in homozygosity or compound heterozygosity with another classic mutation. The L320V mutation has previously been found in two subjects with congenital bilateral absence of the vas deferens, with no other symptoms ${ }^{37}$; to our knowledge, no other phenotypical description of this mutation has been published.

On the other allele (allelic segregation was confirmed by analysis of the parents), L997F (c.2991G $>$ C) is a conservative aminoacidic substitution: both amino acids are nonpolar. Because the position of L997F is in the second membrane spanning domain-ninth transmembrane segment, both amino acids fit the hydrophobic transmembrane environment. The R117L (c.350G $>\mathrm{T}$ ) was found in four subjects on the same allele as L997F (as assessed by parents' analysis); it is a nonconservative 


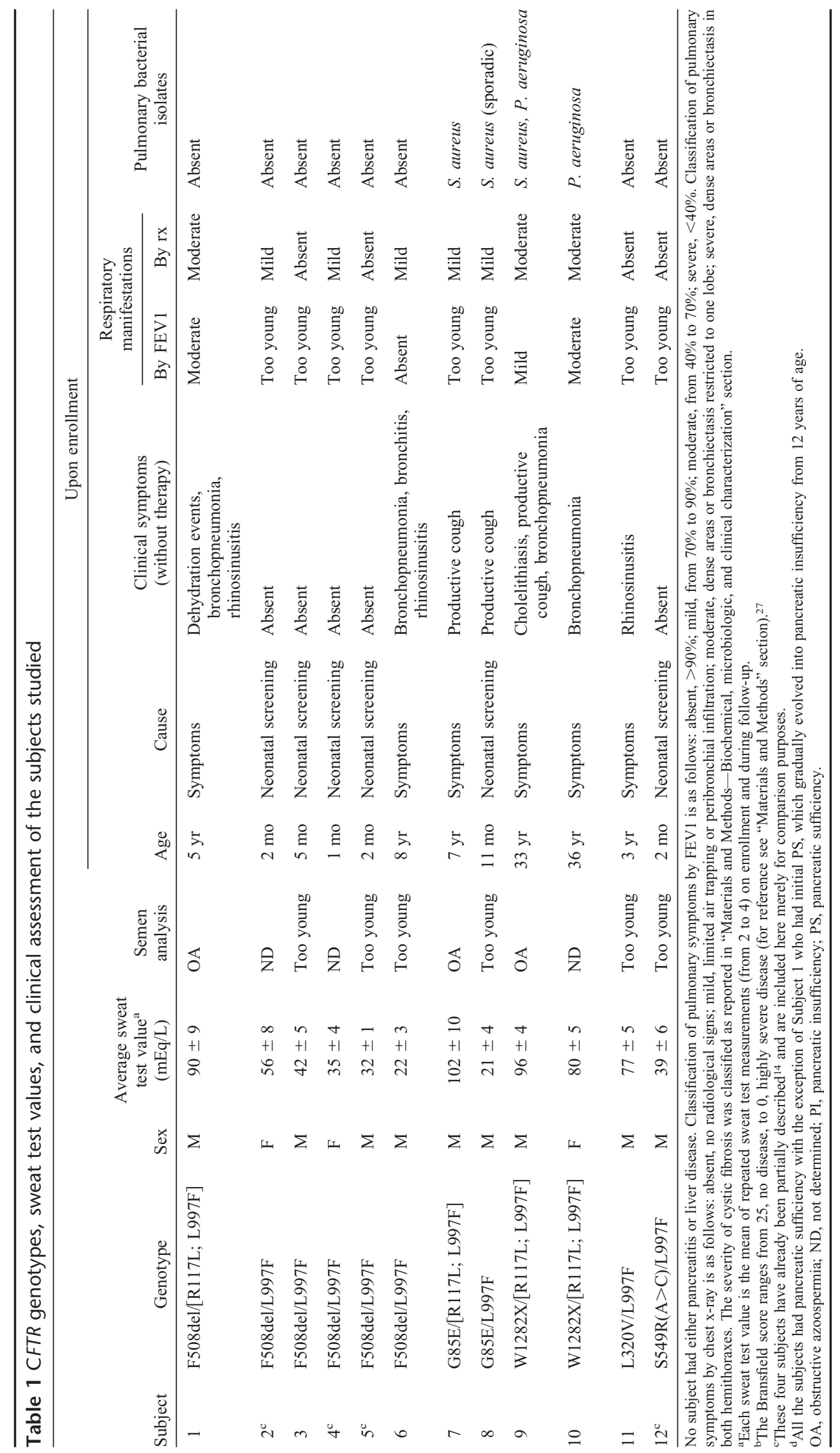




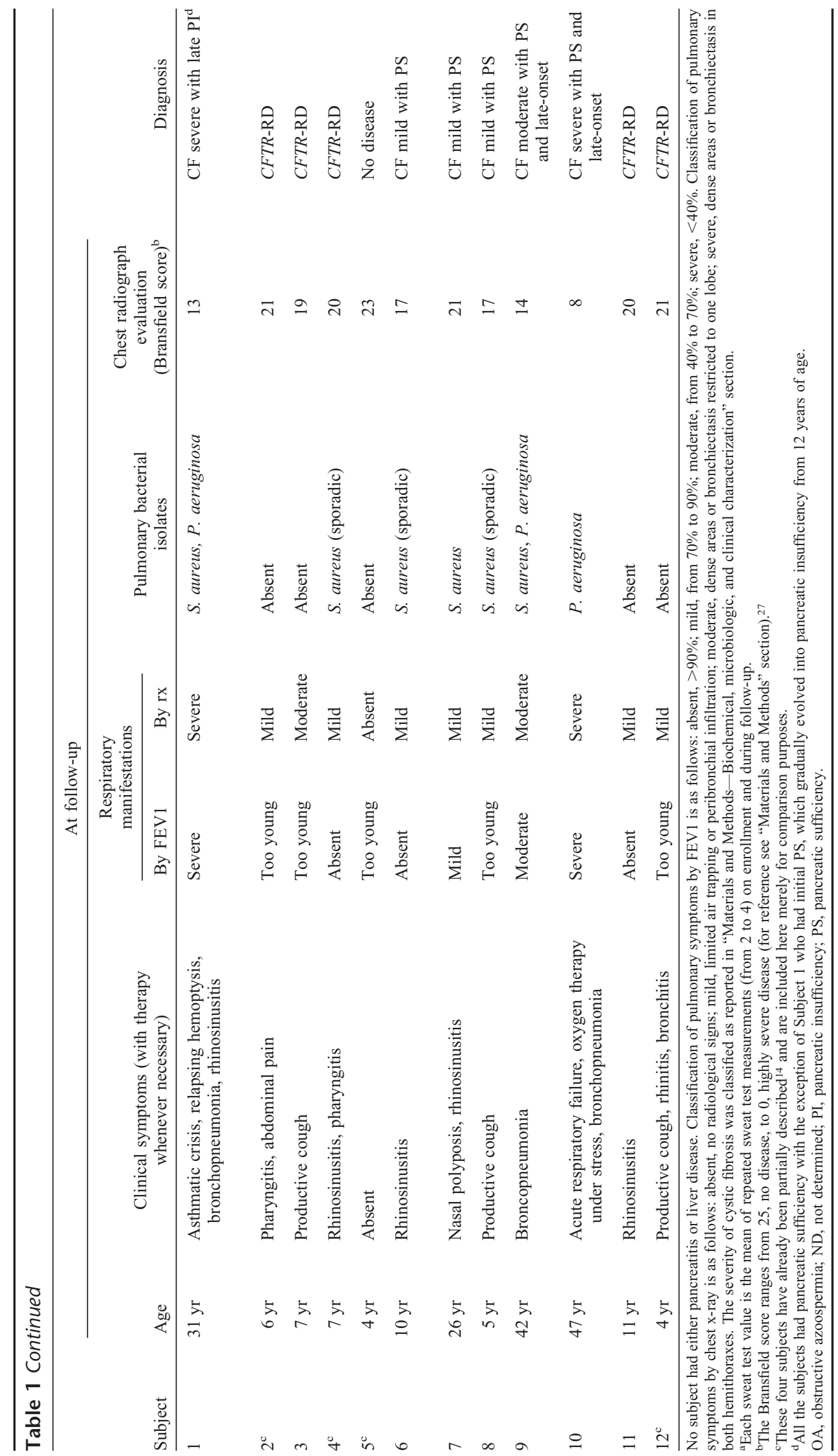


substitution: a positively charged amino acid is substituted by a nonpolar one. Because the position of R117L is in the first extracellular loop exposed to the aqueous extracellular environment, the substituting amino acid will have a lower fit than the original one. Both L997 and R117 aminoacidic residues are completely conserved in the 10 species analyzed.

Among the subjects analyzed, we found the following $(\mathrm{TG})_{m} \mathrm{~T}_{n}$ polymorphic genotypes: $5(\mathrm{TG})_{10} \mathrm{~T}_{9} /(\mathrm{TG})_{10} \mathrm{~T}_{9}, 4$ $(\mathrm{TG})_{10} \mathrm{~T}_{9} /(\mathrm{TG})_{11} \mathrm{~T}_{7}, 2(\mathrm{TG})_{10} \mathrm{~T}_{9} /(\mathrm{TG})_{10} \mathrm{~T}_{7}$, and $1(\mathrm{TG})_{11} \mathrm{~T}_{7} /$ (TG) ${ }_{11} \mathrm{~T}_{7}$. No $(\mathrm{TG})_{12}$ or $\mathrm{T}_{5}$ variant tracts were found. Both the isolated L997F and the [R117L; L997F] complex allele were associated with the $470 \mathrm{~V}$ allele in all subjects.

\section{Genotype-phenotype relationship}

The measurement of the $\mathrm{Cl}^{-}$concentration in the patients sweat is considered as a functional test that measures the in vivo residual ability of CFTR to $\mathrm{Cl}^{-}$transport (the higher the sweat test, the lower the CFTR functionality). The 12 unrelated subjects examined yielded varying sweat test values (Table 1), ranging from 21 to $102 \mathrm{mEq} / \mathrm{L}$, and varying clinical manifestations. In six subjects, the diagnosis of CF was clear since their enrollment and was fully confirmed by follow-up: one had a severe form (Subject 1), another two had a moderate or severe form (Subjects 9 and 10, respectively), while the remaining three had a mild form (Subjects 6, 7, and 8). Subjects 9 and 10 already displayed, on enrollment, a near-complete form of $\mathrm{CF}$, which worsened in both cases during follow-up; because of the late age of symptom onset, they were classified as having a moderate or severe form with late-onset, respectively. A further five subjects (Subjects 2, 3, 4, 11, and 12) were classified as having CFTR-RD, generally with symptoms that were mild on enrollment but worsened during follow-up, without ever reaching a full diagnosis of even mild CF. One subject (Subject 5), who did not display any clinical symptoms either on enrollment or during follow-up, was classified as not having disease.

If one considers only the first two mutations found, the six subjects with apparently the same F508del/L997F genotype (Table 1, Subjects 1-6) showed highly varying sweat test values, ranging from 22 to $90 \mathrm{mEq} / \mathrm{L}$, as did the two subjects (Subjects 7 and 8) with apparently the same G85E/L997F genotype, who had sweat test values of $102 \mathrm{mEq} / \mathrm{L}$ and 21 $\mathrm{mEq} / \mathrm{L}$, respectively. By contrast, the sweat test values in the two subjects with apparently the same W1282X/L997F genotype (Subjects 9 and 10) were more similar $(96 \mathrm{mEq} / \mathrm{L}$ and 80 $\mathrm{mEq} / \mathrm{L}$, respectively). However, the extended genetic analysis detected the R117L mutation on the same allele as the L997F mutation in Subjects $1,7,9$, and 10, thereby revealing a new complex allele of the CFTR gene (an example of the sequencing analysis is shown in Fig. 1). When the presence of this complex allele of the CFTR gene was taken into account, an excellent agreement between genotype and the sweat test values was found. The four subjects with the [R117L; L997F] complex exon 4

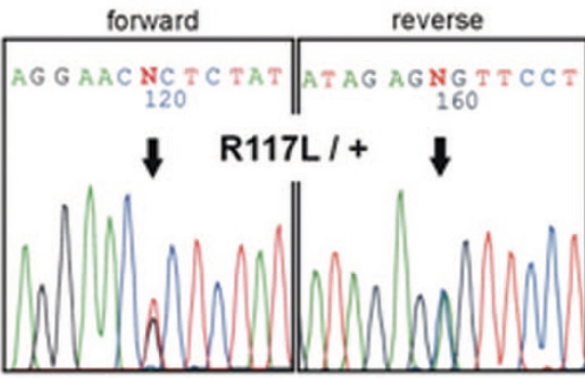

Parent 1 with the [R117L;L997F] complex allele
Subject with the [R117L;L997F] complex allele
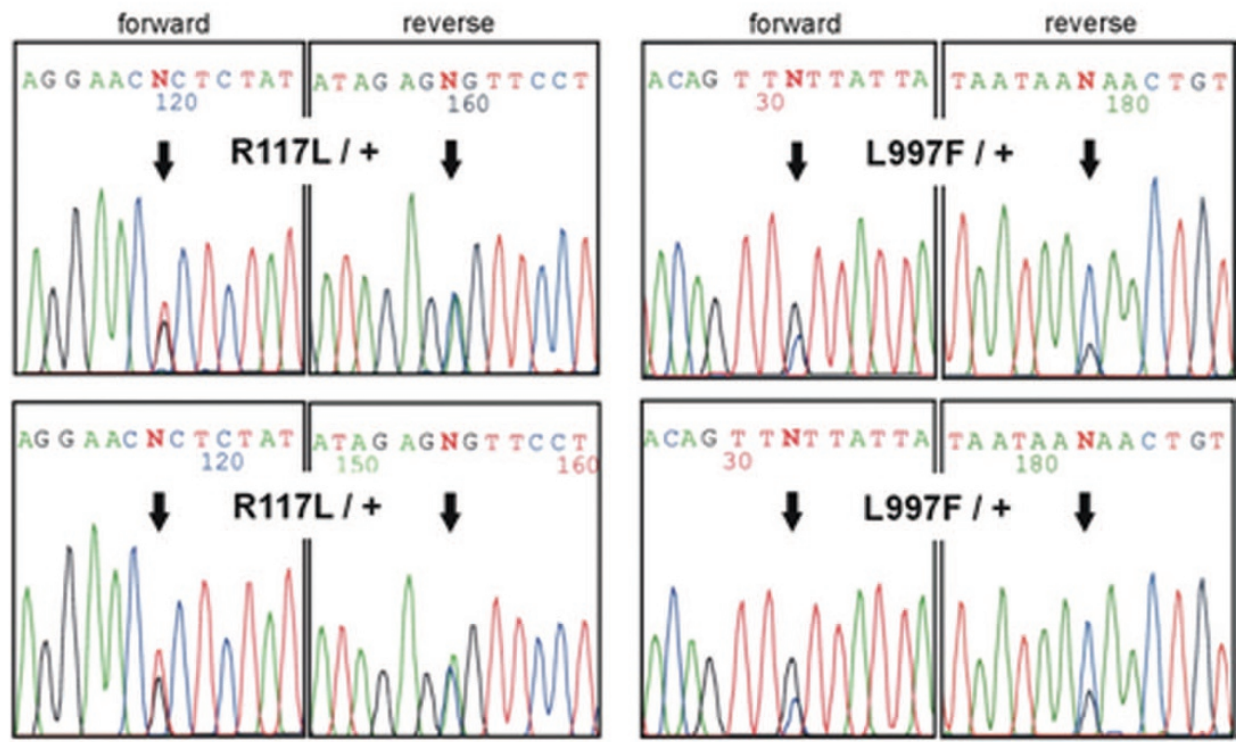

\section{Parent 2 without the complex allele}
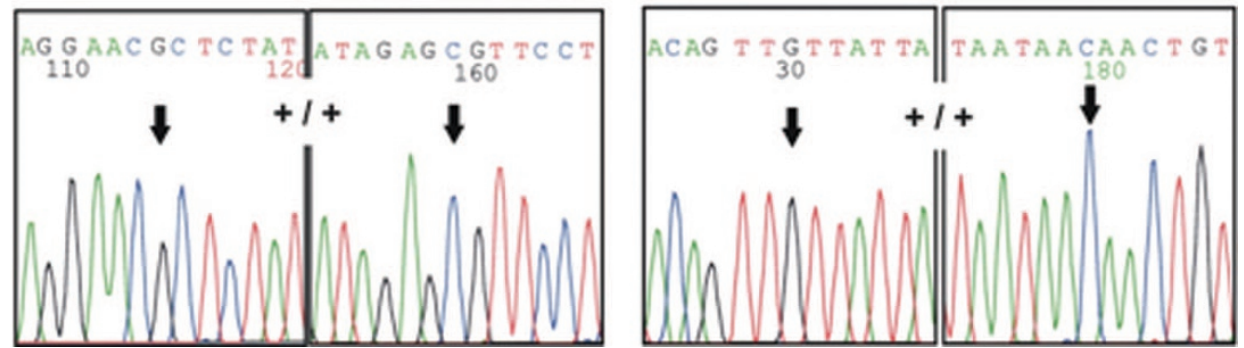

Fig. 1. An example of the complex allele analysis in a family. Some typical sequence electropherograms are shown; the arrows indicate the specific nucleotides. 
allele showed the highest sweat test values among the subjects analyzed, even when otherwise identical genotypes were compared (Subject 1 versus 2-6; Subject 7 versus 8 ). The mean sweat test value in the four subjects with the complex allele (92 $\mathrm{mEq} / \mathrm{L} \pm 9 \mathrm{mEq} / \mathrm{L}$ ) was significantly higher (Student $t$-test $P<$ $0.001)$ than in the eight subjects without the complex allele (41 $\mathrm{mEq} / \mathrm{L} \pm 19 \mathrm{mEq} / \mathrm{L}$ ). A generally good agreement between the presence of the complex allele and clinical outcome was also found. All four subjects with the complex allele were diagnosed as having from mild to severe forms of CF; none of these four subjects was classified as having either CFTR-RD or no disease, as was observed in six of the eight subjects without the complex allele.

The correlation among the complex allele, sweat test values, and clinical outcome is particularly strong if we compare the six subjects with the same F508del mutation on one allele and with or without the complex allele on the other (Table 1). Subject 1, with a F508del/[R117L; L997F] genotype and the highest sweat test value, displayed clinically severe $\mathrm{CF}$ with late pancreatic insufficiency, whereas the five subjects with the F508del/L997F genotype and lower sweat test values displayed either a milder form of $\mathrm{CF}$ (Subject 6) or CFTR-RD (Subjects 2, 3, and 4) or no disease at all (Subject 5). By contrast, the agreement between the presence of the complex allele and clinical outcome was not as good when we compared the two subjects with the same G85E mutation on one allele. Indeed, although the subject with the complex allele (Subject 7) had the highest sweat test value and the subject without the complex allele (Subject 8) had the lowest value in the case series, the clinical outcome in both cases was mild CF. Because the other allele in the two subjects with the W1282X mutation (Subjects 9 and 10) was characterized by the presence of the complex allele, no evaluation of the effect of simple compound heterozygosity was possible. A good correlation was found in these two subjects among the genotype, sweat test, and clinical outcome, as both displayed high sweat test values and clinically moderate or severe CF.

The most varied biochemical and clinical outcome and least clear genotype-phenotype relationship emerged in the eight subjects without the complex allele. Within this group of subjects, Subject 11 was the only one with a pathologic sweat test value; this finding is somewhat surprising as the genotype was expected to be the least severe because it is the only one with fully conservative substitutions on both alleles. Moreover, despite the relatively high sweat test value, the final diagnosis in this subject was of CFTR-RD. The five subjects with the F508del/L997F genotype, which were expected to be more severe than the L320V/L997F genotype, represent a paradigm for variability. Indeed, no pathologic sweat test values were found in these subjects; the two subjects with the borderline sweat test values were diagnosed as having CFTR-RD (Subjects 2 and 3); of the three subjects with the negative sweat test, one was diagnosed as having mild CF (Subject 6), whereas the other two had a CFTR-RD (Subject 4) and no disease (Subject 5). Two other subjects were diagnosed as having either mild CF (Subject 8) or CFTR-RD (Subject 12) despite the negative sweat test, the subject with mild CF displaying a lower sweat test value than the subject with the CFTR-RD.

None of the subjects had either pancreatitis (excluding the involvement of the SPINK1 gene ${ }^{20}$ ) or liver disease. All the subjects had pancreatic sufficiency with the exception of Subject 1; pancreatic sufficiency in this subject gradually evolved into pancreatic insufficiency from the age of 12 years.

\section{DISCUSSION}

Few CFTR complex alleles have been found to date, probably owing above all to the lack of a systematic experimental search. In vivo findings and, in some cases, in vitro functional characterizations have been reported for [F508C; S1251N], ${ }^{38}$ [R347H; D979A], ${ }^{39,40}[\mathrm{R} 74 \mathrm{~W} ; \mathrm{D} 1270 \mathrm{~N}],{ }^{41}$ [G628R; S1235R], ${ }^{42,43}$ [M470V; S1235R], ${ }^{42}[\mathrm{~S} 912 \mathrm{~L} ; \mathrm{G} 1244 \mathrm{~V}],{ }^{44}\left[\mathrm{R} 117 \mathrm{H} ;(\mathrm{TG})_{m} \mathrm{~T}_{n}\right],{ }^{45-47}$ $\left[\mathrm{R} 117 \mathrm{C} ;(\mathrm{TG})_{m} \mathrm{~T}_{n}\right]{ }^{46}\left[\mathrm{~S} 1235 \mathrm{R} ;(\mathrm{TG})_{m} \mathrm{~T}_{5}\right],{ }^{48}[\mathrm{G} 576 \mathrm{~A} ; \mathrm{R} 668 \mathrm{C}],{ }^{10,49}$ [V562I; A1006E], ${ }^{49}$ [R352W; P750L], ${ }^{49}$ [1198_1203del TGGGCT; 1204G >A], ${ }^{49}$ [V754M; CFTRdele3_10,14b_16], ${ }^{50}$ and [F508del; I1027T]. ${ }^{51}$ These complex alleles have been found in patients with either CF or CFTR-RD, although more often in the former. Isolated inheritance results in CFTR-RD, but CF is manifest if both are present in cis. Both in vivo and in vitro studies have also highlighted cases in which there is one main mutation with the phenotypical effect that is worsened by a second mutation, which may even be a neutral variant when isolated, as occurs for F508C, ${ }^{38}$ R74W, ${ }^{41}$ S912L, ${ }^{44}$ and M470V. ${ }^{42}$ However, different effects have also been described, as in the case of the two M470 and R1235 variants, which give rise to a hyperactive CFTR when present on different alleles but have a suppressive effect when combined on the same allele. ${ }^{42}$ In addition, the finding of complex alleles in CFTR-RD seems to suggest that a second CFTR mutation may even lead to a partial reversion of the phenotype. ${ }^{43}$ Indeed, in a reduced number of complex alleles, the effect of the second mutation may partially correct the functional defect, thereby lessening the phenotypical effect, as has been demonstrated for the R553Q mutation in the [F508del; R553Q] complex allele by in vivo $^{52}$ and in vitro ${ }^{53}$ studies and for the R553M mutation in the [F508del; R553M] complex allele by an in vitro study. ${ }^{53}$ A milder phenotypical effect has also been demonstrated for the [R334W; $\mathrm{R} 1158 \mathrm{X}]^{54}$ and [-102T; S549R(T $\left.\left.>\mathrm{G}\right)\right]^{55}$ complex alleles if compared with alleles carrying, respectively, isolated R1158X or S549R(T $>\mathrm{G})$.

Both L997 and R117 aminoacidic residues are highly conserved in the 10 species analyzed, which seems to suggest some evolutionary pressure. L997F is the only known mutation of the 997 CFTR amino acid. ${ }^{37}$ As reported earlier, when isolated, L997F can cause either CFTR-RD (although not CF) or no disease at all. ${ }^{6-19,22,23}$ The conservative nature of the L997F substitution (both residues are hydrophobic) in the CFTR second membrane spanning domain-ninth transmembrane segment may constitute the molecular basis for the limited effect of such an isolated L997F. Five different CFTR mutations of the 117 CFTR amino acid are known: R117C, R117G, R117H, R117L, and R117P. ${ }^{37}$ All these mutations have previously been reported to be more likely to cause CFTR-RD than CF. ${ }^{13,37,46,56}$ However, R117H and R117C have been shown to yield high sweat test values and $C F$, even severe, if cis-acting with the $\mathrm{T}_{5}$ variant tract in CFTR intron 8. ${ }^{45,46}$ If we bear in mind that the $\mathrm{pH}$ range of airway surface fluid is $\mathrm{pH}$ 6.7-7.0, ${ }^{57,58}$ these mutations of the R117 CFTR residue represent both conservative and nonconservative substitutions.

In particular, the $\mathrm{R} 117 \mathrm{~L}$ is a nonconservative substitution that changes the basic residue to a hydrophobic residue. However, in the absence of other mutations or variants on the same allele, evaluation of the hydrophobic character of the R117 substitution fails to correlate with phenotypical alterations. A possible molecular mechanism for the reduced effect of the isolated R117L mutation may be that only 11 of the 15 amino acids that constitute the first extracellular loop domain in which the $\mathrm{R} 117$ residue is located have a charged or a polar side chain, whereas the other four are hydrophobic.

L997F was found in compound heterozygosity with another CFTR mutation in six subjects (four of whom had the complex allele) with CF (mild or severe), in five subjects with CFTR-RD, and in one asymptomatic subject. A good correlation between genotype, sweat test values, and clinical phenotype was found in the four subjects with the complex allele. In these four cases, the mild effects of the isolated L997F and R117L mutations 
cumulate in the complex allele with a cis-acting effect, thereby inducing a well-defined, strong effect on both the $\mathrm{Cl}^{-}$transport (producing the highest sweat test values in the entire case series) and clinical outcome, resulting in $\mathrm{CF}$ (from mild to severe). The other eight subjects without the complex allele did not reveal any clear correlation between genotype and phenotype. The high phenotypical variability in these subjects may be explained on at least two levels. The first level is the weak correlation between genotype and CFTR functionality, which clearly emerges in Subjects 2-6, who have the same genotype but very different sweat test values. The second level is the weak correlation between CFTR functionality and clinical outcome, which emerges through several comparisons. One such comparison may be made between the subjects without the complex allele in whom the clinical outcome does not reflect the sweat test values, as in the cases with mild $\mathrm{CF}$ and a negative sweat test (Subjects 6 and 8), and in the case with CFTR-RD and a positive sweat test (Subject 11). Other ways to highlight this effect are to compare subjects either with similar sweat test values but different disease severity (Subjects 4 and 12 versus 5) or with a similar clinical outcome but different sweat test values (Subjects 2, 3, 4, 11, and 12). There may be several sources of variability on both levels. Although the main CFTR function is $\mathrm{Cl}^{-}$transport, it has been demonstrated that it is also involved, either directly or indirectly through interaction with other proteins, in other functions, i.e., the transport of other ions, the bacterial clearance or the immune/inflammatory response ${ }^{2}$; a possibly altered balance between the different CFTR functions, depending on the CFTR mutations, should be taken into account. In the absence of complex alleles, both levels of variability may in general depend on the limited adverse effect of the L997F mutation, which can easily be modified (worsened or improved) by environmental factors and/or modifier genes. ${ }^{5} \mathrm{~A}$ particular class of modifier genes that specifically may influence $\mathrm{Cl}^{-}$levels are the alternative $\mathrm{Cl}^{-}$channels.

Several genes have been proposed as modifier genes ${ }^{5}$ that influence the second level of variability (sweat test $\rightarrow$ clinical outcome). It is these genes that most probably represent, together with complex alleles, the greatest source of variability in CF. However, even at this level, the role of CFTR may be invoked when the sweat test is negative, but the disease is present. Although the L997F mutation seems to exert a limited influence on $\mathrm{Cl}^{-}$transport (possibly owing to the limited impact of this substitution on the pore structure), it may exert greater influence on other CFTR functions, giving rise to the disease despite having a reduced or even no effect on $\mathrm{Cl}^{-}$transport. Moreover, when sweat test values are not correlated with clinical outcome, potential tissue-specific differences in the amount of $\mathrm{Cl}^{-}$transport, and general CFTR malfunction, must be taken into account. In some cases, the level of $\mathrm{Cl}^{-}$in the sweat may not be proportional to the CFTR functionality in the lung (and other organs), although only in the presence of specific CFTR mutations. In such cases, there is little correlation between the clinical outcome and the sweat test.

As no mutational scan technique that detects all the mutations in the CFTR gene exists, the presence of some other undetected complex allele could be hypothesized in the subjects analyzed. However, this seems somewhat unlikely as the methodology of mutational search we used, which scans all the exons, adjacent intronic zones, and 5'-flanking of the CFTR gene, fails to identify only a small proportion of the CFTR mutations $(<3 \%$ - unpublished results). Any mutations that escape this detection are large deletions and completely intronic mutations that may reveal cryptic exons, both of which are heavy molecular lesions that are most likely to simultaneously produce high sweat test values and a severe clinical outcome.
Another source of variability is age. The six subjects enrolled on the basis of symptoms, including all four subjects with the complex allele, were older than the subjects enrolled by neonatal screening, at both the enrollment and, consequently, follow-up. The fact that the clinical outcome was most severe in these six subjects is probably because they were enrolled before the neonatal screening program had started, their inclusion being exclusively symptom based. Consequently, a worsening in the clinical conditions of those subjects enrolled by means of a neonatal screening protocol cannot be ruled out. This consideration is supported by the fact that a late diagnosis of CF was made in two of the subjects with the complex allele (Subjects 9 and 10), the clinical outcome being classified in one of them as moderate (and not severe) exclusively on account of its late presentation. The late presentation of severe clinical symptoms in subjects with genotypes involving L997F mutation thus seems to be possible.

Although the clinical range in both the group with and the one without the complex allele varies, it also overlaps, from CF mild to $\mathrm{CF}$ severe in the former group and from asymptomatic to $\mathrm{CF}$ mild in the latter group. These findings shed light not only on the phenotypical variability in CF subjects with the L997F mutation but also, more generally, on the issue of the genotype-phenotype relationship in this disease. The vast majority of $\mathrm{CF}$ subjects worldwide undergo a mutational protocol that is interrupted after two mutations have been found on different alleles. Our results highlight the need to search for complex alleles whenever apparently identical CFTR-mutated genotypes are found in subjects with a discordant sweat test and clinical outcome who have not undergone a scanning protocol with a high detection rate. In addition, the following practical conclusions may be drawn from these findings. Whenever a L997F mutation is found, the search for the R117L mutation must be undertaken (and vice versa); if the complex allele is found, the onset of CF (in a mild or severe form) with high sweat test value is likely. If the L997F mutation is the only mutation of the allele, the phenotypical results vary to a greater extent: neither mild CF nor CFTR-RD can be ruled out, although the disease may even be absent, irrespective of the sweat test value. Because the late-onset of $\mathrm{CF}$ is possible even in the presence of the complex allele, particularly when L997F is found alone, a prolonged follow-up is recommended.

\section{ACKNOWLEDGMENTS}

This work was supported by Grants from the Regione Lazio 2007 and the Sapienza Università di Roma, Progetti di Università e Ateneo Federato 2007. S.P. was supported by a Grant from the Associazione Laziale Fibrosi Cistica.

The six subjects enrolled by means of neonatal screening were selected by the two screening centers in the Lazio region (Italy): the central laboratory of the Italian Red Cross of Rome (four subjects) and the screening laboratory of the Department of Experimental Medicine, Sapienza University of Rome (two subjects). The initial genetic characterization of the subject with the L320V/L997F genotype was performed by the Regional Reference Center for Rare Diseases, Department of Pediatrics, University Hospital of Padova (Italy).

\section{REFERENCES}

1. O'Sullivan BP, Freedman SD. Cystic fibrosis. Lancet 2009;373:1891-1904.

2. Riordan JR. CFTR function and prospects for therapy. Annu Rev Biochem 2008;77:701-726.

3. Dequeker E, Stuhrmann M, Morris MA, et al. Best practice guidelines for molecular genetic diagnosis of cystic fibrosis and CFTR-related disordersupdated European recommendations. Eur J Hum Genet 2009;17:51-65.

4. Zhang L, Button B, Gabriel SE, et al. CFTR delivery to $25 \%$ of surface epithelial cells restores normal rates of mucus transport to human cystic fibrosis airway epithelium. PLoS Biol 2009; 7:e1000155. 
5. Collaco JM, Cutting GR. Update on gene modifiers in cystic fibrosis. Curr Opin Pulm Med 2008;14:559-566.

6. Fanen P, Ghanem N, Vidaud M, et al. Molecular characterization of cystic fibrosis: 16 novel mutations identified by analysis of the whole cystic fibrosis conductance transmembrane regulator (CFTR) coding regions and splice site junctions. Genomics 1992;13:770-776.

7. Bombieri C, Luisetti M, Belpinati F, et al. Increased frequency of CFTR gene mutations in sarcoidosis: a case/control association study. Eur J Hum Genet 2000;8:717-720.

8. Bombieri C, Benetazzo M, Saccomani A, et al. Complete mutational screening of the CFTR gene in 120 patients with pulmonary disease. Hum Genet 1998;103:718-722.

9. Girodon E, Cazeneuve C, Lebargy F, et al. CFTR gene mutations in adults with disseminated bronchiectasis. Eur J Hum Genet 1997;5:149-155.

10. Pignatti PF, Bombieri C, Marigo C, Benetazzo M, Luisetti M. Increased incidence of cystic fibrosis gene mutations in adults with disseminated bronchiectasis. Hum Mol Genet 1995;4:635-639.

11. Castellani C, Benetazzo MG, Tamanini A, Begnini A, Mastella G, Pignatti P. Analysis of the entire coding region of the cystic fibrosis transmembrane regulator gene in neonatal hypertrypsinaemia with normal sweat test. $J$ Med Genet 2001;38:202-205.

12. Gomez Lira M, Benetazzo MG, Marzari MG, et al. High frequency of cystic fibrosis transmembrane regulator mutation L997F in patients with recurrent idiopathic pancreatitis and in newborns with hypertrypsinemia. Am J Hum Genet 2000;66:2013-2014.

13. Padoan R, Bassotti A, Seia M, Corbetta C. Negative sweat test in hypertrypsinaemic infants with cystic fibrosis carrying rare CFTR mutations. Eur $J$ Pediatr 2002;161:212-215.

14. Narzi L, Ferraguti G, Stamato A, et al. Does cystic fibrosis neonatal screening detect atypical CF forms? Extended genetic characterization and 4-year clinical follow-up. Clin Genet 2007;72:39-46.

15. Castellani C, Gomez Lira M, Frulloni L, et al. Analysis of the entire coding region of the cystic fibrosis transmembrane regulator gene in idiopathic pancreatitis. Hum Mutat 2001;18:166.

16. Maire F, Bienvenu T, Ngukam A, Hammel P, Ruszniewski P, Levy P. Frequency of CFTR gene mutations in idiopathic pancreatitis. Gastroenterol Clin Biol 2003;27:398-402.

17. Conklin L, Zeitlin PL, Cuffari C. Cystic fibrosis presenting as recurrent pancreatitis in a young child with a normal sweat test and pancreas divisum: a case report. J Med Case Reports 2008;2:176.

18. Casals T, Bassas L, Egozcue S, et al. Heterogeneity for mutations in the CFTR gene and clinical correlations in patients with congenital absence of the vas deferens. Hum Reprod 2000;15:1476-1483.

19. Girodon E, Sternberg D, Chazouilleres O, et al. Cystic fibrosis transmembrane conductance regulator (CFTR) gene defects in patients with primary sclerosing cholangitis. J Hepatol 2002;37:192-197.

20. Gomez Lira M, Bonamini D, Castellani C, et al. Mutations in the SPINK1 gene in idiopathic pancreatitis Italian patients. Eur J Hum Genet 2003;11:543-546.

21. Masson E, Le MC, Levy P, et al. Co-inheritance of a novel deletion of the entire SPINK1 gene with a CFTR missense mutation (L997F) in a family with chronic pancreatitis. Mol Genet Metab 2007;92:168-175.

22. Derichs N, Schuster A, Grund I, et al. Homozygosity for L997F in a child with normal clinical and chloride secretory phenotype provides evidence that this cystic fibrosis transmembrane conductance regulator mutation does not cause cystic fibrosis. Clin Genet 2005;67:529-531.

23. Stanke F, Ballmann M, Bronsveld I, et al. Diversity of the basic defect of homozygous CFTR mutation genotypes in humans. J Med Genet 2008;45:47-54.

24. Bombieri C, Giorgi S, Carles S, et al. A new approach for identifying non-pathogenic mutations. An analysis of the cystic fibrosis transmembrane regulator gene in normal individuals. Hum Genet 2000;106:172-178.

25. Gibson LE, Cooke RE. A test for concentration of electrolytes in sweat in cystic fibrosis of the pancreas utilizing pilocarpine by iontophoresis. Pediatrics 1959;23:545-549.

26. Gullo L, Graziano L, Babbini S, Battistini A, Lazzari R, Pezzilli R. Faecal elastase 1 in children with cystic fibrosis. Eur J Pediatr 1997;156:770-772.

27. Brasfield D, Hicks G, Soong S, Peters J, Tiller R. Evaluation of scoring system of the chest radiograph in cystic fibrosis: a collaborative study. AJR Am J Roentgenol 1980;134:1195-1198.

28. Miller MB, Gilligan PH. Laboratory aspects of management of chronic pulmonary infections in patients with cystic fibrosis. J Clin Microbiol 2003;41:4009-4015.

29. Castellani C, Southern KW, Brownlee K, et al. European best practice guidelines for cystic fibrosis neonatal screening. J Cyst Fibros 2009;8:153-173.

30. Farrell PM, Rosenstein BJ, White TB, et al. Guidelines for diagnosis of cystic fibrosis in newborns through older adults: Cystic Fibrosis Foundation consensus report. J Pediatr 2008;153:S4-S14.

31. World Health Organization. Laboratory manual for the examination of human semen and sperm-cervical mucus interaction, 4th ed. New York: Cambridge University Press; 1999.

32. Grossman PD, Bloch W, Brinson E, et al. High-density multiplex detection of nucleic acid sequences: oligonucleotide ligation assay and sequencecoded separation. Nucleic Acids Res 1994;22:4527-4534.

33. Lucarelli M, Narzi L, Piergentili R, et al. A 96-well formatted method for exon and exon/intron boundary full sequencing of the CFTR gene. Anal Biochem 2006;353:226-235.

34. Lucarelli M, Grandoni F, Rossi T, Mazzilli F, Antonelli M, Strom R. Simultaneous cycle sequencing assessment of (TG)m and Tn tract length in CFTR gene. Biotechniques 2002;32:540-547.

35. Castellani C, Cuppens H, Macek M Jr, et al. Consensus on the use and interpretation of cystic fibrosis mutation analysis in clinical practice. $J$ Cyst Fibros 2008;7:179-196.

36. Frossard PM, Hertecant J, Bossaert Y, Dawson KP. Genotype-phenotype correlations in cystic fibrosis: clinical severity of mutation S549R(T->G). Eur Respir J 1999;13:100-102.

37. Consortium for CF Genetic Analysis, 2006. Available at: http://www. genet.sickkids.on.ca/cftr/. Accessed June 24, 2010.

38. Kalin N, Dork T, Tummler B. A cystic fibrosis allele encoding missense mutations in both nucleotide binding folds of the cystic fibrosis transmembrane conductance regulator. Hum Mutat 1992;1:204-210.

39. Hojo S, Fujita J, Miyawaki H, Obayashi Y, Takahara J, Bartholomew DW. Severe cystic fibrosis associated with a deltaF508/R347H + D979A compound heterozygous genotype. Clin Genet 1998:53:50-53

40. Clain J, Fritsch J, Lehmann-Che J, et al. Two mild cystic fibrosis-associated mutations result in severe cystic fibrosis when combined in cis and reveal a residue important for cystic fibrosis transmembrane conductance regulator processing and function. $J$ Biol Chem 2001;276:9045-9049.

41. Fanen P, Clain J, Labarthe R, et al. Structure-function analysis of a doublemutant cystic fibrosis transmembrane conductance regulator protein occurring in disorders related to cystic fibrosis. FEBS Lett 1999;452:371-374.

42. Wei L, Vankeerberghen A, Jaspers M, Cassiman J, Nilius B, Cuppens H. Suppressive interactions between mutations located in the two nucleotide binding domains of CFTR. FEBS Lett 2000;473:149-153.

43. Mercier B, Verlingue C, Lissens W, et al. Is congenital bilateral absence of vas deferens a primary form of cystic fibrosis? Analyses of the CFTR gene in 67 patients. Am J Hum Genet 1995;56:272-277.

44. Clain J, Lehmann-Che J, Girodon E, et al. A neutral variant involved in a complex CFTR allele contributes to a severe cystic fibrosis phenotype. Hum Genet 2005;116:454-460.

45. Kiesewetter S, Macek M Jr, Davis C, et al. A mutation in CFTR produces different phenotypes depending on chromosomal background. Nat Genet 1993;5:274-278

46. Massie RJ, Poplawski N, Wilcken B, Goldblatt J, Byrnes C, Robertson C. Intron-8 polythymidine sequence in Australasian individuals with $\mathrm{CF}$ mutations R117H and R117C. Eur Respir J 2001;17:1195-1200.

47. Peckham D, Conway SP, Morton A, Jones A, Webb K. Delayed diagnosis of cystic fibrosis associated with R117H on a background of 7T polythymidine tract at intron 8. J Cyst Fibros 2006;5:63-65.

48. Feldmann D, Couderc R, Audrezet MP, et al. CFTR genotypes in patients with normal or borderline sweat chloride levels. Hum Mutat 2003;22:340.

49. McGinniss MJ, Chen C, Redman JB, et al. Extensive sequencing of the CFTR gene: lessons learned from the first 157 patient samples. Hum Genet 2005; $118: 331-338$

50. Niel F, Legendre M, Bienvenu T, et al. A new large CFTR rearrangement illustrates the importance of searching for complex alleles. Hum Mutat 2006;27:716-717.

51. Fichou Y, Genin E, Le MC, Audrezet MP, Scotet V, Ferec C. Estimating the age of CFTR mutations predominantly found in Brittany (Western France). J Cyst Fibros 2008;7:168-173.

52. Dork T, Wulbrand U, Richter T, et al. Cystic fibrosis with three mutations in the cystic fibrosis transmembrane conductance regulator gene. Hum Genet 1991;87:441-446.

53. Teem JL, Berger HA, Ostedgaard LS, Rich DP, Tsui LC, Welsh MJ. Identification of revertants for the cystic fibrosis delta F508 mutation using STE6-CFTR chimeras in yeast. Cell 1993;73:335-346.

54. Duarte A, Amaral M, Barreto C, Pacheco P, Lavinha J. Complex cystic fibrosis allele R334W-R1158X results in reduced levels of correctly processed mRNA in a pancreatic sufficient patient. Hum Mutat 1996;8:134-139.

55. Romey MC, Guittard C, Chazalette JP, et al. Complex allele [-102T> $\mathrm{A}+\mathrm{S} 549 \mathrm{R}(\mathrm{T}>\mathrm{G})]$ is associated with milder forms of cystic fibrosis than allele S549R(T $>$ G) alone. Hum Genet 1999;105:145-150.

56. Daudin M, Bieth E, Bujan L, Massat G, Pontonnier F, Mieusset R. Congenital bilateral absence of the vas deferens: clinical characteristics, biological parameters, cystic fibrosis transmembrane conductance regulator gene mutations, and implications for genetic counseling. Fertil Steril 2000;74:1164-1174.

57. Jayaraman S, Song Y, Vetrivel L, Shankar L, Verkman AS. Noninvasive in vivo fluorescence measurement of airway-surface liquid depth, salt concentration, and pH. J Clin Invest 2001;107:317-324.

58. Thiagarajah JR, Song Y, Haggie PM, Verkman AS. A small molecule CFTR inhibitor produces cystic fibrosis-like submucosal gland fluid secretions in normal airways. FASEB J 2004;18:875-877. 